“ (C) 2016 IEEE. Personal use of this material is permitted. Permission from IEEE must be obtained for all other uses, in any current or future media, including

reprinting/republishing this material for advertising or promotional purposes, creating new collective works, for resale or redistribution to servers or lists, or reuse of any copyrighted component of this work in other works." 


\title{
Generating Localized Waves from Independently Addressable Pulse Driven Arrays
}

\author{
Richard W. Ziolkowski \\ Department of Electrical and Computer Engineering, University of Arizona, Tucson, AZ, USA \\ Global Big Data Technologies Centre, University of Technology Sydney (UTS), Sydney, Australia \\ ziolkowski@ece.arizona.edu
}

\begin{abstract}
After a brief historical perspective on the origins and developments of space-time coupled solutions of the wave equation, the so-called localized waves (LWs), my presentation will emphasize how they can be and have been launched from independently addressable pulse driven (IAPD) arrays. These IAPD arrays are characterized by each element having the possibility to be driven with an individualized time domain waveform. Several acoustic and electromagnetic LW solutions, the IAPD arrays than can launch them, and the near-field and extended far-field region behaviors characterizing the localized beams launched by them, as well as the experiments that have verified these effects, will be discussed.
\end{abstract}

Keywords-Arrays, localized waves, pulses, space-time

\section{INTRODUCTION}

Focused wave modes (FWMs) were introduced by Dr. Jim Brittingham in 1983 [1]. These space-time coupled solutions of the Maxwell equations had provocative properties, i.e., they were electromagnetic (EM) pulses that were localized in space and time and did not disperse as they propagated. Because their development occurred during the Star Wars era, the nomenclature of the time discussing related solutions and their properties was associated with "weapons" related terms, i.e., EM Missiles [2]; acoustic and EM Bullets [3]; and acoustic (ADEPTs) and electromagnetic (EDEPTS) directed energy pulse trains [4].

When dealing with superpositions of these space-time solutions [4], [5], it made more sense to link the tern localized waves (LWs) with their pencil-beam behavior in contrast to the standard sums of highly non-localized plane waves. I began to emphasize the concept of LWs when the independently addressable pulse driven (IAPD) array theory, simulations, and experiments were reported [6]-[9]. Moreover, as we learned how to represent sub-luminal, luminal and super-luminal LWs in phase space [10], [11], LWs became the preferred terminology to denote these solutions as they were found to have some form of localization in space-time and/or phase space, as well as their projections onto physical or other spaces.

Other classes of LWs also began to appear at the time. These included the so-called diffraction-free or non-diffracting Bessel beams [12], complex pulsed beams [13], [14], and nondiffracting $\mathrm{X}$ waves [15].

\section{FINITE ENERGY LWs}

It was shown in [16] that the focus wave mode is an exact solution of the three-dimensional wave equation: $\left\{\nabla^{2}-\partial_{c t}^{2}\right\} \Phi(x, y, z, t)=0$, and can be represented naturally with a bi-directional decomposition [17]:

$$
\Phi(x, y, z, t)=\exp \{i k(z+c t)\} F(x, y, z-c t) .
$$

It leads a Schrödinger equation in the retarded characteristic variable: $\left\{\nabla_{\perp}^{2}+4 i k \partial_{z-c t}\right\} F=0$, which identifies the FWM as a modulated, moving, axi-symmetric Gaussian pulse:

$$
\Phi_{F W M, k}(\rho, z-c t, z+c t)=e^{i k(z+c t)} \frac{e^{-k\left(x^{2}+y^{2}\right) /\left[z_{0}+i(z-c t)\right]}}{4 \pi i\left[z_{0}+i(z-c t)\right]}
$$

where $\rho=\sqrt{x^{2}+y^{2}}$. This bi-directional, characteristic variable solution approach was used to obtain LW fields in many wave environments, even the quantum domain as solutions of the Klein-Gordon and Schrödinger equations [18].

As with all non-spreading solutions like the Bessel beam, the FWMs contain infinite energy. To overcome this issue, it was realized that, in analogy with plane wave representations, superpositions of LWs were possible [16], such as the FWM representation of solutions of the wave equation:

$$
\Phi(z, y, z, t)=\int_{0}^{\infty} d k A(k) \Phi_{F W M, k}(\rho, z-c t, z+c t)
$$

which yielded, for example, the splash pulse [16]. By properly selecting the spectrum $A(k)$, it was recognized that finite energy LWs can be obtained [5], such as the modified power spectrum (MPS) pulse solution of the wave equation:

$$
\Phi_{M P S}(x, y, z, t)=\frac{1}{4 \pi i[a+i(z-c t)]} \frac{\exp [-b s(\rho, z-c t, z+c t) / \beta]}{[b+s(\rho, z-c t, z+c t) / \beta]^{\alpha}}
$$

where the bi-directional term $s(\rho, z-c t, z+c t)=$ $-i(z+c t)+\rho^{2} / a+i(z-c t)$. The corresponding electromagnetic MPS solution was also given in [5]. 


\section{INDEPENDENTLY ADDRESSABLE PULSE DRIVEN ARRAYS}

It was recognized that LWs could be physically realized from an array of radiating elements [6]-[9]. The idea basically follows a Huygens representation, i.e., if one knows the solution at every point on a plane, then one can define the appropriate currents to generate it in a preferred hemisphere. Because a LW is a space-time field, appropriate pulses have to be radiated from each element on that plane corresponding to the spatial dependence of the LW. Hence, to generate LWs, one needs an independently addressable pulse driven (IAPD) array. The received time signal at one spatial point is then a linear superposition of the time signals generated by the entire set of the IAPD array elements.

An important aspect of the analysis of these pulse driven arrays is the recognition that the frequency behavior of each radiating element impacts the transmitted and received signals in the time domain, e.g., electrically small antennas act as time derivative devices. Moreover, the propagation from the near to the far field also involves a time derivative. Several possible time domain outcomes of the complete transmit-receive process are thus possible and some are indicated in Fig. 1.

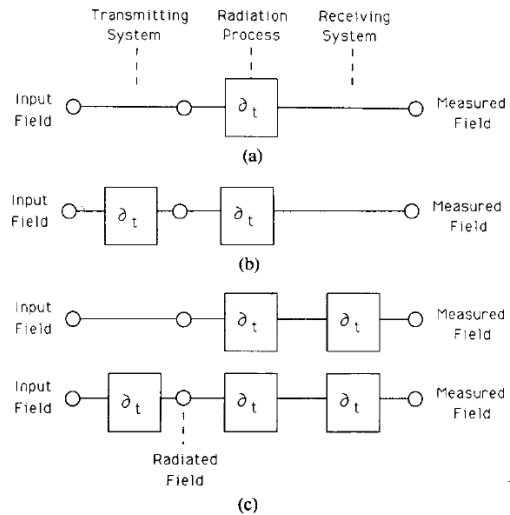

Fig. 1. The output signal of a transmit-receive system depends on the frequency response of its individual antenna elements. It can be proportional to multiple time derivatives of the input signal. (a) One, (b) two, and (c) three time derivative cases.

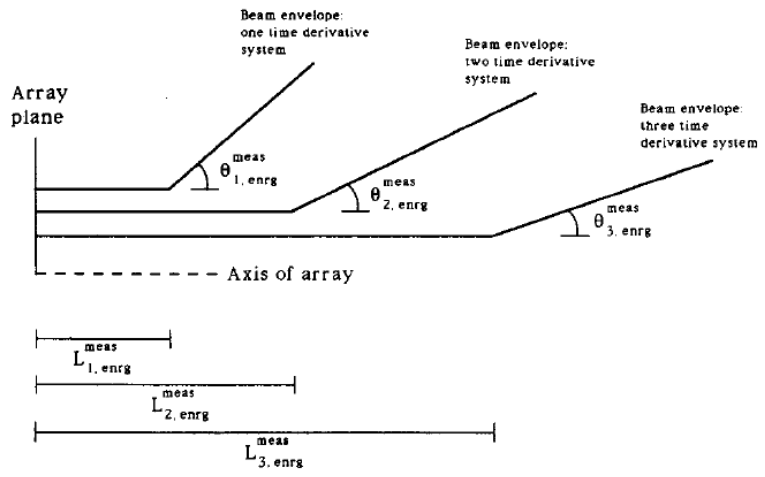

Fig. 2. Diffraction affects the higher order coherence properties of an IAPD transmit-receive array response more slowly than its lower orders. The energy spreading of the beams associated with one, two and three time derivative systems is depicted.
It was recognized [6]-[9] that the resulting beam fields would have dramatically different near-to-far field behaviors depending on the radiating elements. In particular, the higher order time-derivative systems would have extended near field regions. This behavior is depicted in Fig. 3. Moreover, again because of the time domain nature of the diffraction process, the spreading of the energy and power aspects of the launched LW beams would be different. Water tank experiments performed at Lawrence Livermore National Laboratory verified the possibility of launching the acoustic MPS LW solution [6] and its extended near-field distance properties [7]. Examples of LW solutions, how they were launched from IAPD arrays, and the successful confirmation of the predicted LW beam properties will be included in my presentation.

\section{REFERENCES}

[1] J.N.Brittingham, "Focus wave modes in homogeneous Maxwell's equations: transverse electric mode,” J. Appl. Phys., vol. 54, pp. 11791189, Mar. 1983.

[2] T. T. Wu, "Electromagnetic missiles," J. Appl. Phys., vol. 57, pp. 23702373, Apr. 1985.

[3] H. E. Moses and R. T. Prosser, "Initial conditions, sources, and currents for prescribed time-dependent acoustic and electromagnetic fields in three dimensions, Part I: The inverse initial value problem. Acoustic and electromagnetic 'bullets,' expanding waves, and imploding waves," IEEE Trans. Antennas Propag., vol. AP-34, pp. 188-196, Feb. 1986.

[4] R. W. Ziolkowski, "Localized transmission of wave energy," Microwave and Particle Beam Sources and Directed Energy Concepts, Proc. SPIE Conference, vol. 1061, pp. 395-402, Los Angeles, CA, January 1989.

[5] R. W. Ziolkowski, "Localized transmission of electromagnetic energy," Phys. Rev. A, vol. 39, pp. 2005-2033, Apr. 1989.

[6] R. W. Ziolkowski, D. K. Lewis, and B. D. Cook, "Evidence of localized wave transmission," Phys. Rev. Lett., vol. 62, pp. 47-150, Jan. 1989.

[7] R. W. Ziolkowski and D. K. Lewis, "Verification of the localized wave transmission effect," J. Appl. Phys., vol. 68, pp. 6083-6086, Dec. 1990.

[8] R. W. Ziolkowski, "Localized wave physics and engineering," Phys. Rev. A, vol. 44, pp. 3960-3984, Sep. 1991.

[9] R. W. Ziolkowski, "Properties of electromagnetic beams generated by ultra-wide bandwidth pulse-driven arrays," IEEE Antennas and Propagat., vol. 40, pp. 888-903, Aug. 1992.

[10] R. Donnelly and R. W. Ziolkowski, "A method for constructing solutions of homogeneous partial differential equations: localized waves," Proc. Roy. Soc. London A, vol. A437, pp. 673-692, Jun. 1992.

[11] R. Donnelly and R. W. Ziolkowski, "Designing localized waves," Proc. Roy. Soc. London A, vol. A440, pp. 541-565, Mar. 1993.

[12] J. Durnin, J. J. Miceli, Jr., and J. H. Eberly, "Diffraction-free beams," Phys. Rev. Lett., vol. 58, pp. 1499-1501, Apr. 1987.

[13] E. Heyman and L. B. Felsen, "Propagating pulsed beam solutions by complex source parameter substitution," IEEE Trans. Antennas Propag., vol. AP-34, pp. 1062-1065, Aug. 1986.

[14] E. Heyman and B. Z. Steinberg, "Spectral analysis of complex source pulsed beams," J. Opt. Soc. Am. A, vol. 4, pp. 473-480, Mar. 1987.

[15] J. Lu and J. F. Greenleaf, "Nondiffracting X waves - Exact solutions to free-space scalar wave equation and their finite aperture realizations," IEEE Trans. Ultrason. Ferroelectr. Freq. Control, vol. 39, pp. 19-31, Jan. 1992.

[16] R. W. Ziolkowski, "Exact solutions of the wave equation with complex source locations," J. Math. Phys., vol. 26, pp. 861-863, Apr. 1985.

[17] I. M. Besieris, A. M. Shaarawi, and R. W. Ziolkowski, "A bidirectional travelling plane representation of exact solutions of the scalar wave equation," J. Math. Phys., vol. 30, pp. 1254-1269, Jun. 1989.

[18] A. M. Shaarawi, I. M. Besieris, and R. W. Ziolkowski, "A novel approach to the synthesis of nondispersive wave packet solutions to the Klein-Gordon and the Dirac equations," J. Math. Phys., vol. 31(11), pp. 2511-2519, 1990. 